\title{
TUMOR DETECTION IN MEDICAL IMAGING: A SURVEY
}

\author{
Ed-Edily Mohd. Azhari ${ }^{1}$, Muhd. Mudzakkir Mohd. Hatta ${ }^{1}$, Zaw Zaw Htike ${ }^{1}$ and \\ Shoon Lei $\mathrm{Win}^{2}$ \\ ${ }^{1}$ Department of Mechatronics Engineering, IIUM, Kuala Lumpur, Malaysia \\ ${ }^{2}$ Department of Biotechnology Engineering, IIUM, Kuala Lumpur Malaysia
}

\begin{abstract}
A tumor also known as neoplasm is a growth in the abnormal tissue which can be differentiated from the surrounding tissue by its structure. A tumor may lead to cancer, which is a major leading cause of death and responsible for around $13 \%$ of all deaths world-wide. Cancer incidence rate is growing at an alarming rate in the world. Great knowledge and experience on radiology are required for accurate tumor detection in medical imaging. Automation of tumor detection is required because there might be a shortage of skilled radiologists at a time of great need. This paper reviews the processes and techniques used in detecting tumor based on medical imaging results such as mammograms, $x$-ray computed tomography $(x$-ray CT) and magnetic resonance imaging (MRI). We find that computer vision based techniques can identify tumors almost at an expert level in various types of medical imagery assisting in diagnosing myriad diseases.
\end{abstract}

\section{KEYWORDS}

Tumor Detection, Medical Imaging, Computer Vision, Machine Learning

\section{INTRODUCTION}

Statisitcally tumors are the second cause of cancer-related deaths in children (both males and females) whose are under the age of 20 and in males whose age 20 to 39 [1-5,27]. This disease is also the fifth leading cause of cancer-related deaths in females ages 20-39. This facts increase the importance of the researches on the tumor detection and this will present the opportunity for doctors to help save lives by detecting the disease earlier and perform necessary actions. Varieties of image processing techniques are available to be applied on various imaging modalities for tumor detection that will detect certain features of the tumors such as the shape, border, calcification and texture. These features will make the detection processes more accurate and easier as there are some standard characteristics of each features for a specific tumor [6-7].

All tumors will start small and grow with time. As they grow, they will become more conspicuous and increase the probability of showing their characters. A person with tumor usually has certain symptoms and this will bring that person to a physician. From this, they will be able to detect the smallest possible symptomatic malignant (cancerous) tumors that is in early stage and the smallest possible asymptomatic tumors in the screening process. Basically, there are many factors that can influence the appearance of tumors in different kind of processed images despite some common features of malignancies because of variation in the type of tissue and tumor. For large tumor, characteristic features often to be found while in small tumors, these features of malignancy do not appear to be many and some of them might represent themselves by secondary effects such as distortion in its architecture [8-12]. 
In the case of a suspected tumor, "a doctor may perform a neurologic exam to determine if the patient's senses, reflexes, mental status and memory are working normally. The doctor may also order imaging tests, including computed tomography (CT) or magnetic resonance imaging (MRI) of the brain, to pinpoint the tumor and show its size."[13]

Imaging technology has progressed immensely in recent years. Different kind of images can be produced just by a single-click and different image processing techniques can be done to these images to study in detail about it. Machine learning technology has also been used to solve numerous real-world problems especially in medicine [20-42]. Image-based tumor detection uses one or more algorithms as the primary modeling. Some can detect edges, some can detect shapes while others can detect other features. With advances in camera sensing and computational technologies, advances in tumor detection using these features have been an extremely active research area in the intelligent medical community. Clearly, recent researches and trials have extremely help in advancing diagnostic tools for medical purposes but still, the fact that gains in survival need to be achieved by better diagnostic tools $[14,15]$.

\subsection{Magnetic Resonance Imaging (MRI)}

MRI is commonly used in the medical field for detection and visualization of details in the internal structure of the body. It is basically used to detect the differences in the body tissues which have a considerably better technique as compared to computed tomography [23]. Thus, this technique become a special technique especially for the brain tumor detection and cancer imaging [23]. Basically, for comparison, CT uses ionizing radiation while MRI uses strong magnetic field to align the nuclear magnetization that follows by changes the alignment of the magnetization by radio frequencies that can be detected by the scanner. The signal produced can be further processed later to gain extra information of the body [23].

This paper provide a review of image-based tumor detection. The authors then review imagebased tumor detection, commenting on techniques applied for color detection and shape detection. They provide their insights and perspectives on future research directions in image-based tumor detection.

\section{BACKGROUND ON TUMOR DETECTION}

Nowadays, brain tumor has become one of the main cause for increasing mortality among children and adults [23]. Based on some researches, it has been found that the number of people suffering and dying from brain tumors has been increased to 300 per year during past few decades [23]. Figure 1 shows the incidence of brain tumor in various age groups.

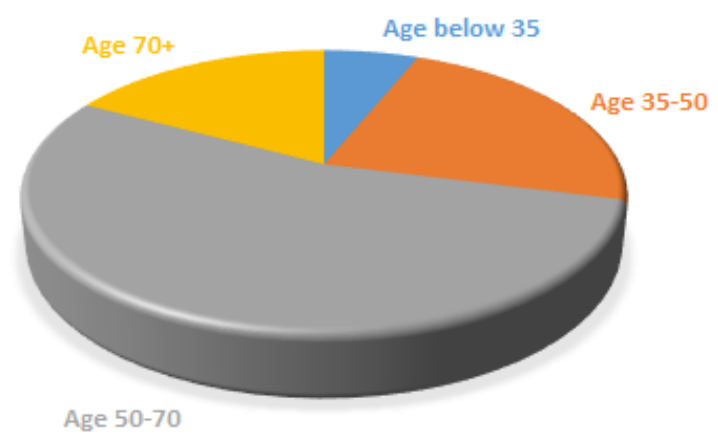

Figure 1: Brain tumor age distribution 
Figure 2 shows the 5-year survival rates over the past three decades. It shows that the survival rates have improved with the advancement in imaging and diagnosis technology.

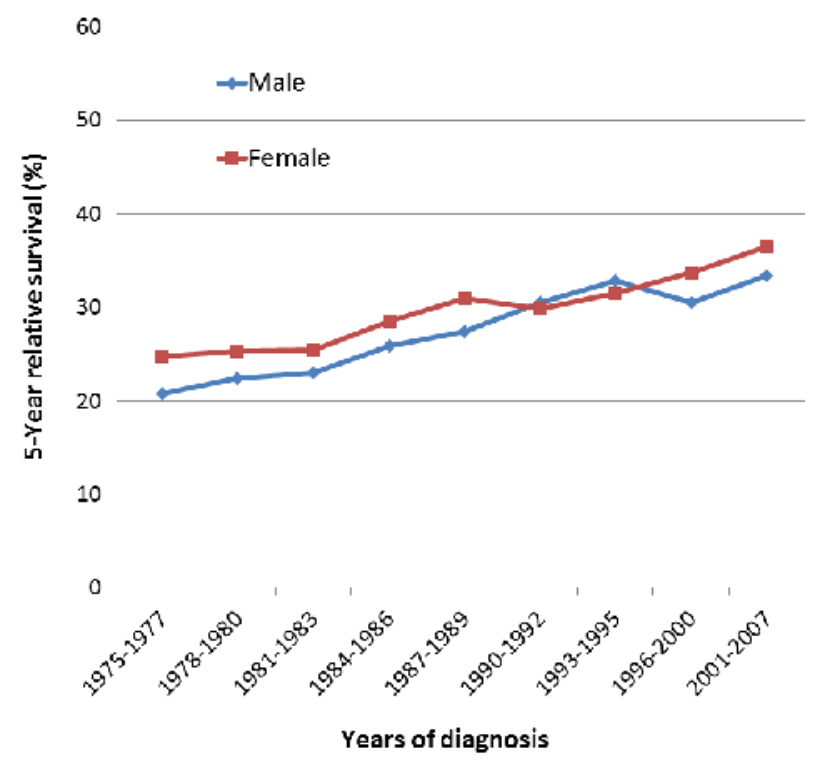

Figure 2: 5-Year brain tumor survival rate over years [24]

According to Nagalkar and Asole [16], CT-Scan technique usually used for monitoring the images of damaged brain part. The images of the CT Scans are shown in the form of gray scale images as the equipment for CT scans support this form of image color and for easy detection of tumor from the image [16]. For example, in the parietal section of the head scanned using CT scans, the Cerebrum part is shown in the form of the gray color while the veins and arteries parts in the form of creamish white color [16]. Any clotting that exist in the brain that show any kind of damage can be detected as dark gray in color. The process of extraction of parameters are basically like taking out per pixel information and then plotting it [16]. For an image obtained from CT-Scan, the images are shown in this manner; tumor appears white and brain damaged cells shown in black color, thus the binary values of the pixel showing the brain damaged cells are 0 and showing the tumor are 1, thus by extraction process, further analysis can be done such as checking and plotting in MATLAB [16]. The patient with damaged brain can be differentiated from normal patient by using this technique. In addition, tumor can also be detected clearly based on the image results [16].

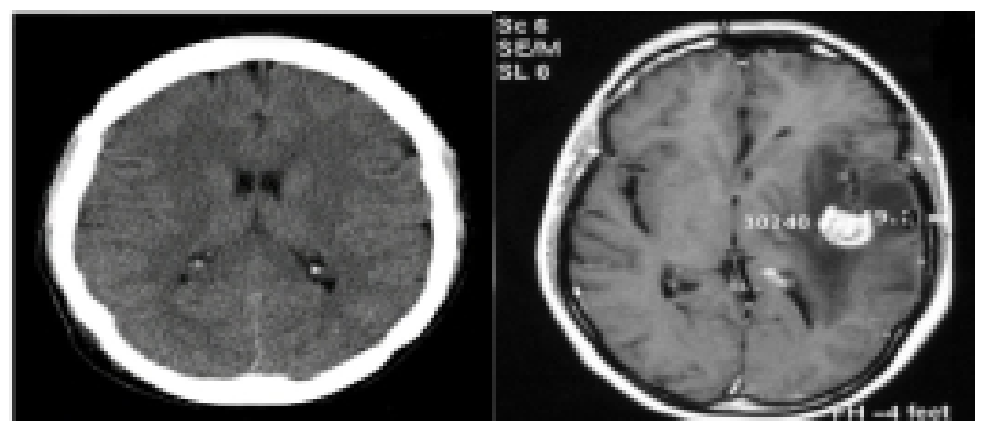

Figure 3: Brain CT Scan image a) Normal patient b) Tumor patient [16] 


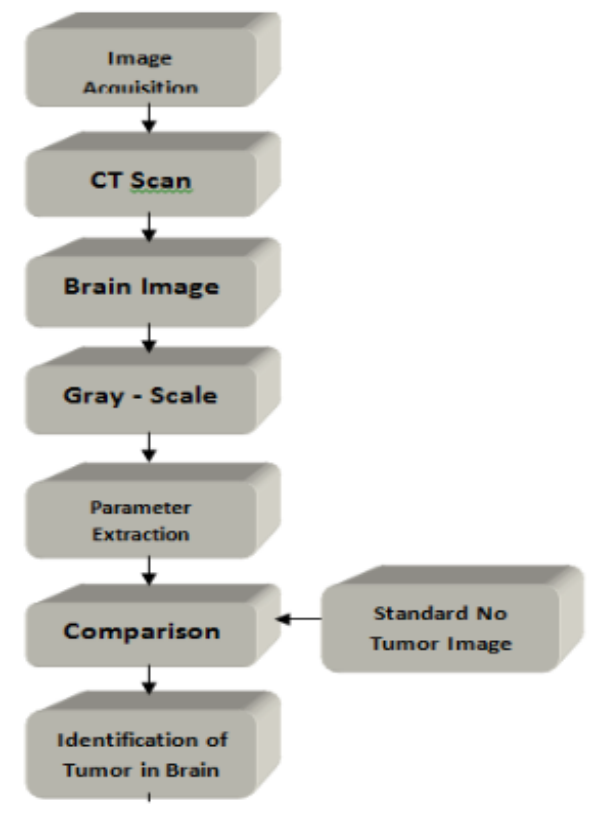

Figure 4: Basic flowchart to determine existence of tumor [16]

Additionally, this technique has been practiced to determine tumor patient's response to treatment since long time ago. The radiologist has made series of cross-sectional diameter measurements for indicator lesions purposes by using axial, incremental CT image data. Later, these measurements will be compared with the previous measurement scans [25]. Nevertheless, the measurement of lesion diameter does not represent the exact assessment of tumor size due to some factors, such as:

\section{Irregular lesions}

The lesions that grow other than sphere shape may not adequately represented by diameters' changes;

\section{Different measurement between inter-observer and intra-observer}

Referred to the image selection used for the measurement and the location of lesion boundary;

\section{Different levels of scanning results collected from various diagnoses}

The lesions may not be captured exactly at the same spot from one diagnosis to another. Hence, it affects the lesion's image in which causes comparisons between examinations becoming more difficult.

On the other hand, with the ability to provide single breath-hold scans and overlapping reconstructions, the spiral CT is capable to improve the image lesions at reproducible levels from different diagnosis [25]. Even so, these lesions' measurements are still a subjective task and yet open for variability as described in previous factors (a) and (b). What follows is a summary of several approaches to the problems involved in nodule detection. Therefore, the researchers have divided these approaches into two groups of methods which are [25]:

(a) The process of detecting the pulmonary vasculature in order to help the detection of pulmonary nodules (if vasculature is successfully removed from the image, then only nodules should remain). 
International Journal of Advanced Information Technology (IJAIT) Vol. 4, No. 1, February 2014

(b) The process of detecting objects in the CT image volume to distinguish between nodules and other structures.

\section{Segmentation of Vascular Structures For Detection of NODULES [25]}

According to McNitt-Gray et al. [25], Croisille et al. [1] came up with a three-dimensional analysis technique to diagnose pulmonary vasculature. Their analysis technique has helped radiologists in detecting pulmonary nodules at an early stage. In their approach, the sequential images of CT were placed together to form an image volume. Then, after the extraction of vascular tree, the heart was carried out manually [25]. The vessels were then extracted from the remaining volumetric data by using a three-dimensional seeded region growing algorithm. The selection of seed point was carried out specifically by the user from the vascular lumen; all voxels within the user-specified gray level (or Hounsfield Unit (HU) - the normalized measure of attenuation used in CT) range that were six-connected (that is, $\mathrm{x}, \mathrm{y}$, or $\mathrm{z}$ connected) to the seed point were added recursively until the entire vascular tree was segmented. At this stage, the process should identify both the pulmonary arterial and venous trees and once identified, the voxels belonging to the vascular tree were removed from the volumetric data set. Later, the remaining voxel in the data set were examined for pulmonary nodules by a radiologist [25].

\section{Automatic Segmentation of Pulmonary Nodules [25]}

Compared to the previous approach, the second method focuses on the nodule detection, rather than the pulmonary vasculature tree. Even though the vascular tree is not explicitly segmented in these methods, a primary concern remains the ability to distinguish nodules from vessels. According to Giger [3], pulmonary nodules on CT can be detected by using a combination of segmentation and morphological techniques. It analyzed each two-dimensional (2-D) slice individually and then compared candidate nodules in neighboring slices. For each 2-D slice, the first step was to detect the thoracic boundary in the image. It was carried out by obtaining a profile from the center of the image to the edge of the image; this profile was used to determine a section-specific threshold from which the boundaries of connected regions are obtained. However, in the case of multiple regions, each region was analyzed by its location, area, and circularity to determine whether it is truly the thorax or another object.

Meanwhile, feature analysis is used to identify the tumors separate from the blood vessels as they cannot be reliably distinguished on the basis of CT number such as by thresholding. The following features were calculated for each candidate region [25]:

- Area: the number of pixels in the candidate region [25]

- Compactness: maximum gray-weighted distance in the candidate region

- Circularity: percentage of occupation inside the candidate region's circumscribed circle

- Position: minimum distance between the centers of the candidate region's circumscribed circle and the lung boundary

Medically, tumors can be recognized by [25]

(a) spherical shape in a 2-D cross-sectional images, whereas vessels running in the scan plane will appear oblong;

(b) the decreasing in thickness of blood vessels when their position approaches the chest wall, whereas tumors are larger; 
(c) shadows contacting the chest wall are usually tumors, because blood vessels at the periphery are too small to be seen in the CT image;

(d) the CT numbers of the blood vessels are usually higher than tumors when the vessels run perpendicular to the scan plane, and

(e) the CT numbers within lung tumors are relatively uniform.

In order to label the anatomical structures in the image data set, image must be matched to the respective objects in the model [25]. This can be done by producing a feature-space to compare both data from image and data from model. The features of image will be compared with the predictions and the best match will be chosen for the object. Some critical information will be contained in the model such as the expected size, shape, and location with respect to other structures. Then, the thresholding process will be done considering the surroundings. The shape of object with different size and shape that are connected will be extracted by morphological process.

With the scarcity of medical knowledge, it is easier to assume and design a system that detects obvious tumors. The paper will need a more detailed analysis in medical terms for the research to proceed in that area of accuracy and flexibility of detecting.

Such detail scanning as in section A and B is to determine lung tumor from CT scan images. The method also involves seeking more information about tumor. However, this paper is only interested in the basic understanding and development of image processing of merely detecting and positioning the tumor.

Part A and B however is important in realizing the limitations of basic image processing in tumor detection mainly because of the extra process to differentiate vascular and pulmonary nodules. These types of tumor (lungs) have a complication to segment out because of its tumor size and similarity with thevascular and pulmonary nodules.

Therefore for this project, methods proposed by Nagalkar and Asole will be taken in consideration.

\section{EDGE DETECTION AND SEgMENTATION}

According to Patil and Balchandra [32], their Matlab algorithm to detect brain tumor given this form of image, involves 2 stages: preprocessing and segmentation and then followed by morphological processes [17].

The structure of their method are as follows [32]:

1. Give MRI image of brain as input.

2. Convert it to gray scale image.

3. Apply high pass filter for noise removal.

4. Apply median filter to enhance the quality of image.

5. Compute threshold segmentation.

6. Compute watershed segmentation.

7. Compute morphological operation.

8. Finally output will be a tumour region.

Dina et al. [17] also agrees with this method. The main focus for image processing in the given format (MRI) is to have an Image segmentation part and an edge detection algorithm. 
Under image segmentation, enhancement and smoothing of the gray scale MRI images is done. Most MRI images encounter Gaussian and impulse noise according to Dina et al.

This method then proposes to use Gaussian smoothing filter to remove Gaussian noise and linear filters to enhance edges:

- $\quad$ Linear filter [17]:

$$
I_{A}(i, j)=I^{*} A=\sum_{h=-n / 2}^{n / 2} \sum_{k=-m / 2}^{m / 2} A(h, k) I(i-h, j-k)
$$

Gaussian filter [17]:

$$
G_{f}(x, y)=\frac{1}{c} \exp \left(\frac{-\left[x^{2}+y^{2}\right]}{2 \sigma^{2}}\right)
$$
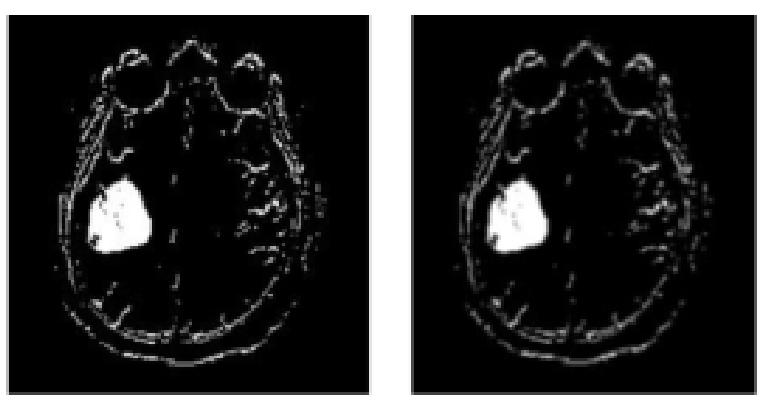

Figure 5: Images after Gaussian filter is applied (left). Use average filter for better processing (right) [17].

The edge detection module is the part of interest. It is to determine the ROIs in the MRI image. This can locate the tumor thus determining its existence and notifying people of its position in the MRI scan.

Dina et al. proposes to use the Canny edge detection. According to Canny [18], the optimal filter that meets all three criteria above can be efficiently approximated using the first derivative of a Gaussian function.
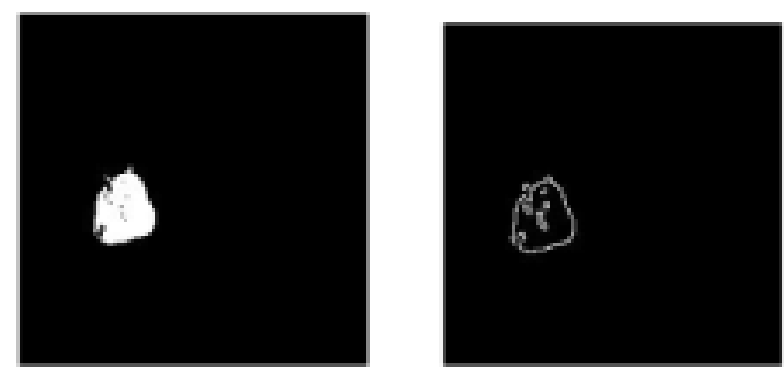

Figure 6: Tumor edge detection with Canny edge detection algorithm [17] 


\section{Conclusion}

The study has identified 2 main processes in determining the existence of tumor. Image segmentation and edge detection. It can be generally concluded that the program will be interested in detecting damaged tissue with a certain intensity of brightness to its grayscale image. The thresholding will detect the damaged tissues. Through the image segmentation process, this research intends to use grayscale converted images of MRIs or CT scans. A series of filters will be used including Gaussian, linear and average filters to remove noise. Through the edge detection process, the research intends to use a canny edge detector which is commonly used in similar environments. The research has also identified several medical limitations and contributions that can be done in future works. This is seen when dealing with the inaccuracy of image processing in separating vascular and pulmonary structures in the human body mainly present in lung tumors.

\section{REFERENCES}

[1] C. Croisille, et al., "Pulmonary nodules: Improved detection with vascular segmentation and extraction with spiral CT", Radiology, 197:397-401, 1995.

[2] T. Tozaki, et al., "Three-dimensional analysis of lung area using thin slice CT images", Medical Imaging Proc SPIE, 2709:2-11, 1996.

[3] M.L. Giger, et al., "Computerized detection of pulmonary nodules in computed tomography images", Investigative Radiology, 29(4): pp. 459-465, 1994.

[4] S. Toshioka, et al., "Computer aided diagnosis system for lung cancer based on helical CT images", Image Processing: KM Hanson, Proc SPIE 3034:pp. 975-984, 1997.

[5] J. Toriwaki, et al., "Fundamental properties of the gray weighted distance transformation", Trans IEICE Japan, J60-D(12):1101-1108, 1977.

[6] D. Cavouras, et al., "Image analysis methods for the solitary pulmonary nodule characterization by computed tomography”. Eur J Radiol 14: 169-172, 1992.

[7] M.F. McNitt-Gray, et al., "Characterization of solitary pulmonary nodules using features extracted from high resolution CT images". Radiology 205(P):395, 1997.

[8] M.F. McNitt-Gray, et al., "The application of image analysis techniques to distinguish benign from malignant solitary pulmonary nodules imaged on CT". Proc SPIE Med Imag 3338, in press, 1998.

[9] Y. Kawata, et al., "Classification of pulmonary nodules in thin section CT images based on shape characterization". IEEE Int Conf Image Proc 3:528-531, 1997.

[10] R.M. Summers, et al., "Automatic detection of endobronchial lesions with virtual bronchoscopy: comparison of two methods". Proc SPIE Med Imag 3338, in press, 1998.

[11] A.P. Reeves, et al., "Characterization of three-dimensional shape and size changes of pulmonary nodules over time from helical CT images”. Radiology 205(P):396.

[12] D.F. Yankelevitz, et al., "Can computer-aided morphologic analysis of solitary pulmonary nodules predict pathology?” Radiology 205(P):529, 1997.

[13] Brain Tumors and Brain Cancer, Retrieved from: http://www.cedars-sinai.edu/Patients/HealthConditions/Brain-Tumors-and-Brain-Cancer.aspx, Retrieved on: 3 December 2013.

[14] American Brain Tumor Association, Facts and statistics, 2010. Retrieved from: http://www.abta.org/sitefiles/pdflibrary/ABTA-FactsandStatistics2010.pdf, Retrieved on: 3 December 2013.

[15] Central Brain Tumor Registry of the United State, 2010, CBTRUS statistical report: Primary brain and central nervous system tumors diagnosed in the United States in 2004-2006. Retrieved from: http://www.cbtrus.org/2010-NPCR-SEER/CBTRUS, Retrieved on: 3 December 2013.

[16] V.J. Nagalkar, S.S Asole, "Brain Tumor Detection Using Digital Image Processing Based On Soft Computing”, Journal Of Signal And Image Processing, Volume 3, Issue 3, pp.-102-105.

[17] D.A. Dahab, et al., "Automated Brain Tumor Detection and Identification Using Image Processing and Probabilistic Neural Network Techniques", International Journal of Image Processing and Visual Communication ISSN2319-1724 Volume 1, Issue 2, October 2012.

[18] H. Demuth, M. Beale, "Neural Network Toolbox User's Guide”, 2000

[19] T. Logeswari, M. Karnan, "An improved implementation of brain tumor detection using segmentation based on soft computing", Journal of Cancer Research and Experimental Oncology Vol. 2(1), 2010, pp 6-14. 
International Journal of Advanced Information Technology (IJAIT) Vol. 4, No. 1, February 2014

[20] Z.Z. Htike, S.L. Win "Recognition of Promoters in DNA Sequences Using Weightily Averaged Onedependence Estimators", Procedia Computer Science, Volume 23, 2013, Pages 60-67, ISSN 18770509.

[21] Z.Z. Htike, S.L. Win "Classification of Eukaryotic Splice-junction Genetic Sequences Using Averaged One-dependence Estimators with Subsumption Resolution”, Procedia Computer Science, Volume 23, 2013, Pages 36-43, ISSN 1877-0509.

[22] Z.Z. Htike, et al., "A Monocular View-Invariant Fall Detection System for the Elderly in Assisted Home Environments", 7th International Conference on Intelligent Environments (IE), 2011, pp.40,46, 25-28 July 2011.

[23] A. Mustaqeem, et al., “An Efficient Brain Tumor Detection Algorithm Using Watershed \& Thresholding Based Segmentation” International Journal of Image, Graphics and Signal Processing, Vol.4, No.10, 2012, pp34-39.

[24] Global cancer statistics: Brain tumor, Retrieved from: http://www.medinfographics.com/cancerstatistics/brain-tumor/, Retrieved on: 5 December 2013.

[25] M.F. McNitt-Gray, et al., "Image-Processing Techniques for Tumor Detection", Edited by R.N. Strickland, CRC Press 2002.

[26] Marker-Controlled Watershed Segmentation, Retrieved http://www.mathworks.com/products/demos/image/watershed/ipexwatershed.html, Retrieved on: 6 December 2013.

[27] Brain Tumor Information, Retrieved from: http://www.abta.org/brain-tumor-information/ Retrieved on: 6 December 2013.

[28] T. Acharya, A.K. Ray, "Image Processing: Principles and Applications", Wiley-Interscience, 2005.

[29] S. Xavierarockiaraj, et al., "Brain Tumor Detection Using Modified Histogram ThresholdingQuadrantApproach", Journal of Computer Applications, Volume V, Issue 1, 2012.

[30] J. Omwoyo, "Canny edge detector", Seminar, Lappeenranta University of Technology, 2007.

[31] N. Liu, M. Lu, "Computational Geometry, Graphs and Applications”, Lecture Notes in Computer Science, 2010.

[32] R.C. Patil, A.S. Bhalchandra, "Brain Tumour Extraction from MRI Images Using MATLAB", International Journal of Electronics, Communication \& Soft Computing Science and Engineering, Volume 2, Issue 1.

[33] Z.Z. Htike, “Can the future really be predicted?", Signal Processing: Algorithms, Architectures, Arrangements, and Applications (SPA), 2013, pp.360-365, 26-28 Sept. 2013.

[34] Z.Z. Htike, "Multi-horizon ternary time series forecasting", Signal Processing: Algorithms, Architectures, Arrangements, and Applications (SPA), 2013, pp.337-342, 26-28 Sept. 2013.

[35] S.L. Win, et al., "Cancer Recurrence Prediction Using Machine Learning", International Journal of Computational Science and Information Technology (IJCSIT), 2014, Vol. 6, Issue 1, ISSN: 0975 3826.

[36] S.N.A. Hassan, et al., "Vision Based Entomology: A Survey", International Journal of Computer science and engineering Survey (IJCSES), 2014, Vol. 5, Issue 1, ISSN: 0976-2760.

[37] E.M. Azhari, et al., "Brain Tumor Detection and Localization in Magnetic Resonance Imaging", International Journal of Information Technology Convergence and services (IJITCS), 2014, Vol. 4, Issue 1, ISSN 2231-1939.

[38] N.A. Mohamad, et al., "Bacteria Identification from Microscopic Morphology Using Naïve Bayes", International Journal of Computer Science, Engineering and Information Technology (IJCSEIT), 2014, Vol. 4, Issue 1, ISSN 2231-3117.

[39] S.L. Win, et al., "Cancer Classification from DNA Microarray Gene Expression Data Using Averaged One-Dependence Estimators", International Journal on Cybernetics \& Informatics (IJCI), 2014, Vol. 3, Issue 1, ISSN: 2320-8430.

[40] S.N.A. Hassan, et al., "Vision Based Entomology - How to Effectively Exploit Color and Shape Features", Computer Science \& Engineering: An International Journal (CSEIJ), 2014, Vol. 4, Issue 1, ISSN: 2231-3583.

[41] N.A. Mohamad, et al., "Bacteria Identification from Microscopic Morphology: A Survey", International Journal on Soft Computing, Artificial Intelligence and Applications (IJSCAI), 2014, Vol. 3, Issue 1, ISSN: 2319-1015.

[42] S.L. Win, et al., Cancer Survivability Prediction Using Semi-Naïve Bayesian Models, International Journal on Bioinformatics \& Biosciences, 2014, Vol. 4, Issue 1, ISSN: 1839-9614. 